\title{
The Reuniens and Rhomboid Nuclei Are Required for Acquisition of Pavlovian Trace Fear Conditioning in Rats
}

\author{
Yu-Ju Lin, ${ }^{1}$ Ruei-Jen Chiou, ${ }^{2}$ and ${ }^{D}$ Chun-hui Chang ${ }^{1}$
}

https://doi.org/10.1523/ENEURO.0106-20.2020

${ }^{1}$ Institute of Systems Neuroscience, National Tsing Hua University, Hsinchu 30013, Taiwan and ${ }^{2}$ Department of Anatomy and Cell Biology, School of Medicine, College of Medicine, Taipei Medical University, Taipei 11031, Taiwan

\begin{abstract}
The reuniens $(R e)$ and rhomboid $(R h)$ nuclei (ReRh) of the midline thalamus interconnects the hippocampus (HPC) and the medial prefrontal cortex (mPFC). Several studies have suggested that the ReRh participates in various cognitive tasks. However, little is known about the contribution of the ReRh in Pavlovian trace fear conditioning, a procedure with a temporal gap between the conditioned stimulus (CS) and the unconditioned stimulus (US), and therefore making it harder for the animals to acquire. Because the HPC and mPFC are involved in trace, but not delay, fear conditioning and given the role of the ReRh in mediating this neurocircuitry, we hypothesized that ReRh inactivation leads to a learning deficit only in trace conditioning. In a series of experiments, we first examined the c-Fos expression in male Long-Evans rats and established that the ReRh was recruited in the encoding, but not the retrieval phase, of fear memory. Next, we performed behavioral pharmacology experiments and found that ReRh inactivation impaired only the acquisition, but not the consolidation or retrieval, of trace fear. However, although the ReRh was recruited during the encoding of delay fear demonstrated by c-Fos results, ReRh inactivation in any phases did not interfere with delay conditioning. Finally, we found that trace fear acquired under ReRh inactivation reprised when the ReRh was brought offline during retrieval. Together, our data revealed the essential role of the ReRh in a learning task with temporally discontinuous stimuli.
\end{abstract}

Key words: behavioral pharmacology; nucleus reuniens; rhomboid nucleus; trace fear conditioning

\section{Significance Statement}

The behavior and neurobiology of normal and pathologic fear learning have long been an interest to scientists since malfunction of fear regulation may lead to severe psychiatric disorders. To capture the complex behavior of fear learning, in a rodent model, we studied trace fear conditioning, along with delay procedure, to investigate the recruitment of the reuniens $(R e)$ and rhomboid $(R h)$ nuclei $(R e R h)$ in a task with temporally discontinuous stimuli. We found that the ReRh is involved during the acquisition of trace fear. However, without a functional ReRh during acquisition, retrieval of trace fear is state-dependent and likely generalized. Our study revealed the essential role of the ReRh in different learning procedures of fear conditioning.

\section{Introduction}

Fear is a vital negative emotion that helps organisms cope with potential dangers, avoid further harm, and increase survival chances (LeDoux, 1996). However,

Received March 19, 2020; accepted June 2, 2020; First published June 10, 2020.

The authors declare no competing financial interests. malfunction of fear may lead to mental disorders, such as phobia, panic disorder, and posttraumatic stress disorder (PTSD; Milad et al., 2006; Quirk and Mueller, 2008). Therefore, it is essential to study the psychological

Author contributions: Y.-J.L. and C.-h.C. designed research; Y.-J.L. performed research; R.-J.C. contributed unpublished reagents/analytic tools; Y.-J.L. and C.-h.C. analyzed data; Y.-J.L. and C.-h.C. wrote the paper. 
processes and neurobiological mechanisms of fear learning. Pavlovian fear conditioning is frequently used to study the neuronal bases of fear learning and memory (Rigoli et al., 2016). In this behavioral procedure, animals learn to associate the initially neutral conditioned stimulus (CS), e.g., a tone, with an aversive unconditioned stimulus (US), e.g., a mild footshock. Different temporal arrangements of the stimuli lead to different behavioral procedures: in delay conditioning, the CSs and USs overlap in presentation and co-terminate, while in trace conditioning, the stimuli are separated in time by a "trace" interval (Pavlov, 1927; Raybuck and Lattal, 2014). Trace fear learning resembles human emotional learning in that human learning sometimes involves a temporal gap. Notably, the insertion of a trace interval affects associative learning (Shors et al., 2000). Subjects usually require more trials to acquire trace fear (Pavlov, 1927; Beylin et al., 2001) and the trace CS-US association is weaker, demonstrated by lower freezing levels following trace conditioning (Raybuck and Lattal, 2014). Indeed, this variant of the procedure recruits profoundly different and higher-order neuronal circuitry. The importance of the hippocampus (HPC) and the medial prefrontal cortex (mPFC) in trace conditioning has been repeatedly examined. Bangasser et al. (2006) demonstrated that hippocampal lesions only impaired trace, but not delay, fear conditioning. Indeed, electrophysiology results showed that hippocampal intrinsic excitability and synaptic plasticity are involved in the acquisition of trace fear conditioning (Song et al., 2012). Gilmartin and Helmstetter (2010) showed that pharmacological inactivation or blockade of NMDA receptor-dependent transmission in the MPFC impaired the acquisition of trace fear conditioning. Moreover, active firing of mPFC neurons (Blum et al., 2006; Gilmartin et al., 2013) and changes in intrinsic excitability of amygdalaprojecting mPFC neurons (Song et al., 2015) were demonstrated in trace fear conditioning.

Anatomically, the mPFC does not directly project to the HPC (Laroche et al., 2000; Vertes, 2004), but it can communicate with the HPC through the reuniens $(R e)$ and rhomboid (Rh) nuclei (ReRh) of the midline thalamus (Hoover and Vertes, 2012). Other than the ReRh, the perirhinal cortex (PRC; Witter et al., 2000; Delatour and Witter, 2002) and entorhinal cortex (EC; Burwell and Amaral, 1998; Agster and Burwell, 2009) might also act as relay stations. The ReRh interconnects with the mPFC and the HPC (Vertes et al., 2006) and is necessarily involved in tasks that require coordinated $\mathrm{mPFC}$ and HPC activity. Indeed, inactivation of the ReRh caused deficits

This work was supported by the Ministry of Science and Technology, Taiwan (MOST) Grant 106-2320-B-007-006-MY3 (to C.-h.C.) and by the Higher Education Sprout Project funded by the Ministry of Science and Technology and Ministry of Education in Taiwan.

Correspondence should be addressed to Chun-hui Chang at changch@ life.nthu.edu.tw.

https://doi.org/10.1523/ENEURO.0106-20.2020

Copyright $\odot 2020$ Lin et al.

This is an open-access article distributed under the terms of the Creative Commons Attribution 4.0 International license, which permits unrestricted use, distribution and reproduction in any medium provided that the original work is properly attributed. in a working memory-dependent conditional discrimination task (Hallock et al., 2013), impaired performance in a spatial working memory-specific task (Layfield et al., 2015), and disrupted hippocampal-prefrontal synchrony (Hallock et al., 2016). Relevant to the present study, Ramanathan et al. (2018a) recently demonstrated that inactivation of the ReRh impaired the acquisition of contextual fear memory and led to fear generalization. These studies suggest that the ReRh coordinates the mPFCHPC network, which is important in the establishment and maintenance of the association between stimuli in various cognitive tasks (Cassel et al., 2013; Dolleman-van der Weel et al., 2019). Previous research speculated that the mPFC may modulate memory specificity via its inputs to the ReRh, which in turn signals to both the HPC and back to the MPFC, making the ReRh a critical node in the mPFC-HPC circuitry (Xu and Südhof, 2013).

Given the role of the ReRh in mPFC-HPC interactions, we sought to determine whether the ReRh is involved in different learning phases of trace fear conditioning and hypothesized that recruitment of the ReRh is necessary only for trace, but not delay, fear conditioning. To address our question, we used immunohistochemical approach to quantify c-Fos expression, followed by behavioral pharmacology experiments, to examine the general recruitment and the determinant role of the ReRh in different learning phases (acquisition, consolidation, and retrieval) of trace fear. We also explored whether there is a statedependent effect of the ReRh inactivation as suggested by previous literature in contextual fear conditioning (Ramanathan et al., 2018a). Together, our data revealed the importance of the ReRh during the acquisition of trace fear conditioning.

\section{Materials and Methods}

\section{Subjects}

A total of 180 Long-Evans male rats (initially weighing 200-224 g; National Laboratory Animal Center, NARLabs, Taiwan) were used. All animals were individually housed in the animal facility at National Chiao Tung University under professional animal care staff and on-site veterinarian. Animals were maintained on a 12/12 h light/dark cycle with food and water provided ad libitum. All animals were handled for at least $5 \mathrm{~d}(10 \mathrm{~s} / \mathrm{d})$ before any experimental procedure. All experimental procedures were performed during the light cycle (7 A.M. to 7 P.M.) and followed the guidelines approved by both of National Tsing Hua University and National Chiao Tung University, Institutional Animal Care and Use Committees (IACUC).

\section{Surgical procedures}

Animals were anesthetized with ketamine $(80-100 \mathrm{mg} /$ $\mathrm{kg})$ and xylazine $(8-10 \mathrm{mg} / \mathrm{kg}$ ) and then placed in a stereotaxic apparatus (Stoelting). Core body temperature was maintained at $37^{\circ} \mathrm{C}$ by a temperature-controlled heating pad (CWE). A single 26-gauge stainless steel guide cannula was implanted aiming the Re (relative to bregma: anterior-posterior $-2.3 \mathrm{~mm}$, medial-lateral $+1.9 \mathrm{~mm}$, and dorsal-ventral $-6.5 \mathrm{~mm}$ ) at a $15^{\circ}$ angle to the vertical axis. 
A

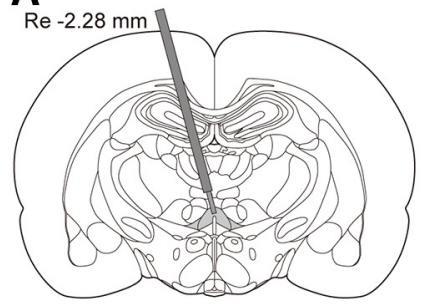

B

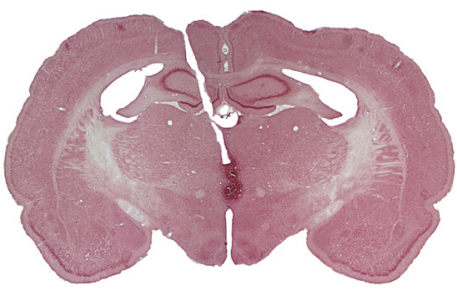

C

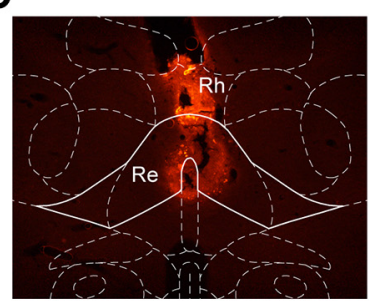

Figure 1. Histology confirmation of this study. A, An illustrative representation showing cannula placement in the ReRh. $\boldsymbol{B}, \mathrm{A}$ Nisslstained coronal section showing the cannula placement in the ReRh. $\boldsymbol{C}, \mathrm{A}$ dark-field image showing diffusion of TMR-X muscimol in the ReRh.

An example of illustrative representation and its Nisslstained coronal section are shown in Figure $1 A, B$. Three anchor screws were mounted and the headstage was fixed with dental acrylic. Carprofen $(5 \mathrm{mg} / \mathrm{kg})$ was subcutaneously injected after surgery and the following $2 \mathrm{~d}$. After surgery, animals were placed in their home cages and monitored until awake. Behavioral procedures were performed at least $5 \mathrm{~d}$ after surgeries. In the meantime, dummies $(1.0 \mathrm{~mm}$ longer than cannula) were changed daily to prevent blockade of the cannulae.

\section{Drug infusions}

Drug infusions were performed at different time points according to our experimental design (experiments 2-5, see below); 33-gauge injectors (extending $1.0 \mathrm{~mm}$ beyond the guide cannula) were attached to polyethylene tubes that were connected to Hamilton syringes located on an infusion pump (Harvard Apparatus). Muscimol $(0.5 \mu \mathrm{g} / \mu \mathrm{l}$; Alfa Aesar) or vehicle (sterile saline) was injected at the rate of $0.25 \mu \mathrm{l} / \mathrm{min}$ for $2 \mathrm{~min}$, followed by another $30 \mathrm{~s}$ for drug diffusion. An example of the diffusion of fluorescentlabeled TMR-X muscimol (Life Technologies) in the ReRh is shown in Figure 1C. Notably, the inactivation was subtotal and was limited to the injection center of the Re and the above Rh, whereas the more lateral portions of the $\mathrm{Re}$ were not affected. The dosage of muscimol was chosen based on earlier studies for similar experimental purposes (Hallock et al., 2013; Layfield et al., 2015). The effect of muscimol lasts $\sim 3-4 \mathrm{~h}$ after injection (Martin, 1991; van Duuren et al., 2007).

\section{Behavioral apparatus}

Four fear conditioning chambers (Med-Associates) were used with two context settings. In Context A, animals were transported to the chambers in cuboids. The doors of the chambers were half open and the chamber lights were on. The fans attached to the chambers were in operation, which also worked as background noise. Additionally, the pans beneath the chambers were filled with $1 \%$ acetic acid, providing a distinct odor. In Context $\mathrm{B}$, animals were transported to the chambers in cylinders and covered with black sheets. The lights in the behavioral room were blurred red, which was a dark surrounding for rats. The doors of the chambers were closed and the chamber lights were off. The fans attached to the chambers were off. There were A-frame inserts and acrylic plates above the grids inside the chambers. Additionally, the pans were filled with $1 \%$ ammonium.

\section{Experimental design}

\section{Experiment 1}

Animals were assigned into four groups: TRACE, DELAY, UNPAIRED, and NoCOND. On day 1 , each group received different conditioning procedure in Context $A$. After a 3-min baseline (BL), each group received a tentrial session of auditory fear conditioning using a 20-s auditory CS (85 dB, $2 \mathrm{kHz})$ and a 2-s footshock US (1.0 mA) with a 240-s intertrial interval (ITI). For TRACE rats, there was a 30-s trace interval (stimulus-free period) between CSs and USs, whereas for DELAY rats, the CSs always co-terminated with the USs. For UNPAIRED rats, CSs and USs were not paired, with the interval in between randomized ranging from 100 to $120 \mathrm{~s}$ (average of $110 \mathrm{~s}$ ). For NoCOND rats, they received CSs only but no USs. To assess fear encoding, the amount of time spent freezing during the $B L$ and the first 18-s CS (to avoid disturbance by the footshock in the DELAY group) was measured and analyzed. On day 2, all rats received three 20 -s CS during the test session in Context B with an ITI of $60 \mathrm{~s}$. The test was performed in a different context from conditioning to minimize contextual fear, and only three CSs were given to ensure that c-Fos expression corresponded to fear retrieval, but not fear extinction (Bouton, 2004). To assess fear retrieval, the amount of time spent freezing during the BL and during the 20-s CS was measured and analyzed.

\section{Experiments 2-4}

Three independent experiments with drug manipulation at different time points were done. For each experiment, a mixed design with between-subject factors of "drug" [muscimol (M) and vehicle (V)/or control (C)] and "group" (TRACE and DELAY) and within-subject factor of "trials" was used, yielding a total of four groups: M-TRACE, V(C)TRACE, M-DELAY, and V(C)-DELAY. Drug infusions were performed according to the experimental design: immediately before conditioning (experiment 2), after conditioning (experiment 3), or immediately before retrieval test (experiment 4). The day before the behavioral experiment, the animals were accustomed to the sound of the infusion pump for $2 \mathrm{~min}$. On day 1, animals were assigned into two groups: trace conditioning (TRACE) and delay conditioning (DELAY). The procedures were the same as in experiment 1 and were performed in Context A. On day 2, all 
animals received a ten 20 -s CS test session in Context B with the ITI of $60 \mathrm{~s}$ to assess fear memory.

\section{Experiment 5}

Animals were assigned into three groups: SAL-SAL, MUS-SAL, and MUS-MUS, and received drug infusions immediately before conditioning and immediately before retrieval test, accordingly. The day before the behavioral experiment, the animals were accustomed to the sound of the infusion pump for 2 min. On day 1, all animals underwent trace fear conditioning procedure the same as described in experiment 1 in Context A. On day 2, all animals received a 1020 -s CS test session in Context $B$ with the ITI of $60 \mathrm{~s}$ to assess fear memory.

\section{Immunohistochemistry}

In experiment 1, half of the animals were killed for tissue processing after conditioning, while the other half tested for memory retrieval and then killed after retrieval test for tissue processing. To capture the time window of maximal c-Fos expression, the animals were killed $90 \mathrm{~min}$ after the behavioral sessions (conditioning or retrieval test). All animals were deeply anesthetized with $\mathrm{CO}_{2}$. Incisions were made within $30 \mathrm{~s}$ after animals left the $\mathrm{CO}_{2}$ chamber and animals were completely unresponsive during the entire perfusion procedure. To optimize the perfusion, a hemostat was used to clamp the descending aorta. All animals were perfused transcardially with $100 \mathrm{ml}$ of saline followed by $100 \mathrm{ml}$ of $4 \%$ paraformaldehyde (PFA) in $0.1 \mathrm{M}$ PBS, serving as a fixative. Brains were removed, postfixed at least $2 \mathrm{~h}$, cryoprotected in $25 \%$ sucrose solution for $24-72 \mathrm{~h}$, and then sectioned coronally $(35 \mu \mathrm{m})$. We performed c-Fos visualization with the standard immunohistochemical procedure. In short, free-floating tissue sections were incubated overnight in a buffer solution with mouse anti-c-Fos antibody (Santa Cruz, catalog \#sc$271243,1: 200)$. The following day after rinsing with buffer several times, sections were incubated in biotinylated donkey anti-mouse IgG (Jackson ImmunoResearch, code \#715-065-151, 1:500) solution, and then Vectastatin ABC Elite reagents (Vector Laboratories), followed by a solution of nickel sulfate and diaminobenzidine (DAB) with hydrogen peroxide to produce a blue-black reaction product within the nucleus of c-Fos+ neurons.

\section{Histology}

To verify cannula placements in experiments $2-5$, brains were collected after the retrieval test and fixed with $8 \%$ PFA in PBS. After $48 \mathrm{~h}$, the brains were transferred to $25 \%$ sucrose in $0.1 \mathrm{~m}$ PBS until saturated. Brains were then sectioned coronally $(60 \mu \mathrm{m})$. The slices were mounted onto subbed slides for standard Nissl staining to confirm the injection sites.

\section{Statistical analysis}

For experiment 1, our analyses focused on the Re only. All c-Fos + neurons within the Re were manually counted with microscopy at three coronal levels: $-1.56,-2.52$, and $-3.48 \mathrm{~mm}$, relative to bregma. The counts in each group were submitted to one-way ANOVA. All significant F-ratios were reported, and after significant F-ratios were obtained, Tukey's HSD post hoc analyses were performed.

All behavioral procedures were recorded (Video Freeze, Med-Associates; sampling at $0.2 \mathrm{~s}$ ), and freezing was defined as consecutively observed movements for $1 \mathrm{~s}$ below the motion threshold (program set at 100). Freezing behavior was measured continuously during all of the behavioral sessions. The percentage of total observations in which freezing occurred at BL and during CSs was calculated. These values were submitted to repeated measures of ANOVA (RM ANOVA). All significant F-ratios were reported, and after significant $F$ ratios were obtained, Tukey's HSD post hoc analyses were performed.

For all ANOVAs, effect sizes were reported as partial $\eta^{2}$ $\left(\eta_{\mathrm{p}}^{2}\right.$; Fritz et al., 2012; Lakens, 2013). As an effect size, $\eta_{\mathrm{p}}^{2}$ values $>0.01,0.06$, and 0.14 are generally interpreted as small, medium, and large effects, respectively (Richardson, 2011). All data were calculated using SPSS (IBM) and presented as mean \pm SEM.

\section{Results}

\section{Experiment 1: $R e$ is generally recruited during the encoding, but not the retrieval, of trace and delay fear memory}

In this experiment, we first established whether the Re is recruited in delay and trace fear conditioning using a 2d procedure (Fig. $2 A$ ). A total of 48 animals were used. On day 1 , animals underwent the conditioning procedure according to their assigned groups ( $n=12$ per group), and then half of the animals in each group was killed. On day 2 , the remaining of the animals ( $n=6$ per group) were tested for fear retrieval in a different context to minimize contextual fear and then killed. The tissues were processed to visualize c-Fos expression in the $R e$ as an index of activation during encoding (day 1 ) and retrieval (day 2) of fear memory.

The behavioral results are summarized in Figure $2 C$, left panel. On day 1, TRACE, DELAY, and UNPAIRED groups exhibited a robust increase in freezing levels toward later trials, whereas NoCOND group exhibited low freezing levels throughout the conditioning session. There were significant main effects of group $\left[F_{(3.44)}=72.24, p<0.001\right.$, $\left.\eta_{\mathrm{p}}^{2}=0.83\right]$ and trials $\left[F_{(10,440)}=86.34, p<0.001, \eta_{\mathrm{p}}^{2}=\right.$ $0.66]$, and a significant interaction between group and trials $\left[F_{(30,440)}=7.07, p<0.001, \eta_{p}^{2}=0.33\right]$. Post hoc comparisons suggested that freezing levels in TRACE, DELAY, and UNPAIRED groups were significantly higher compared with NoCOND group (all $p s<0.001$ ). Moreover, there was no statistical difference in freezing levels between TRACE and DELAY groups $(p=0.89$ ), indicating equivalent acquisition under delay and trace procedures during conditioning.

On day 2, the animals were tested for their fear to the tones. Both DELAY and TRACE groups demonstrated high freezing levels during the CSs, while UNPAIRED and NoCOND groups demonstrated low freezing levels. There were significant main effects of group $\left[F_{(3,20)}=29.06\right.$, $\left.p<0.001, \eta_{p}^{2}=0.81\right]$ and trials $\left[F_{(3,60)}=49.70, p<0.001\right.$, 
A

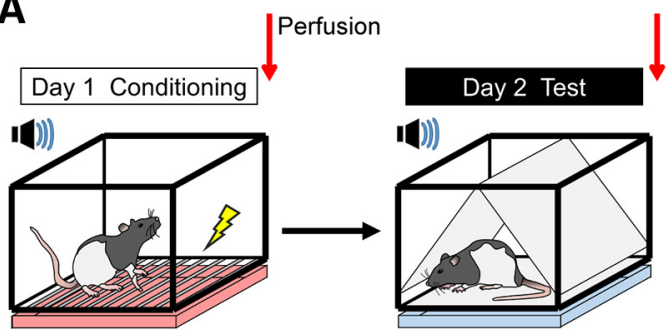

C

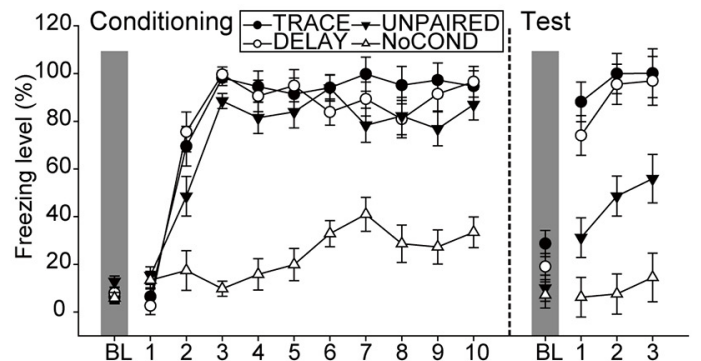

D
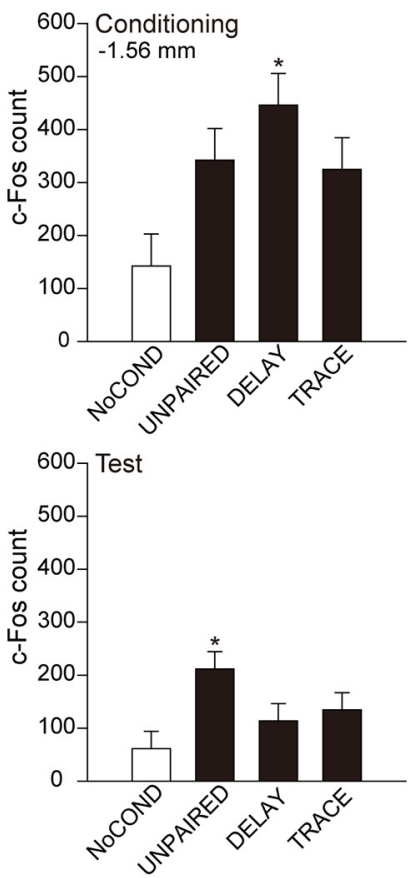

B

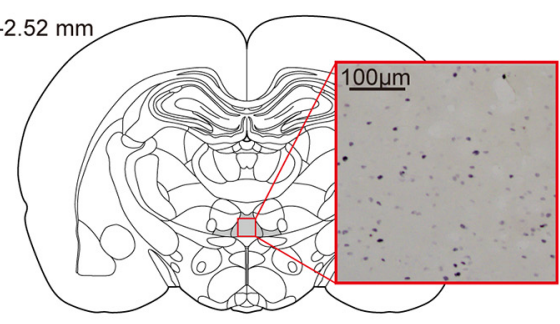

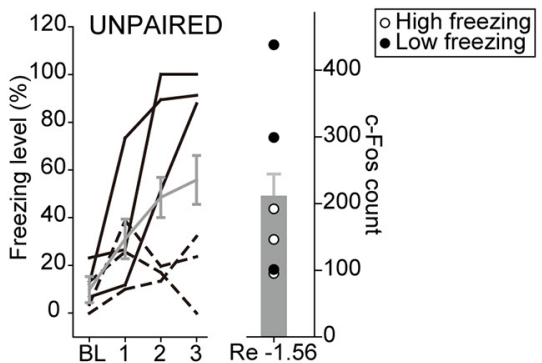
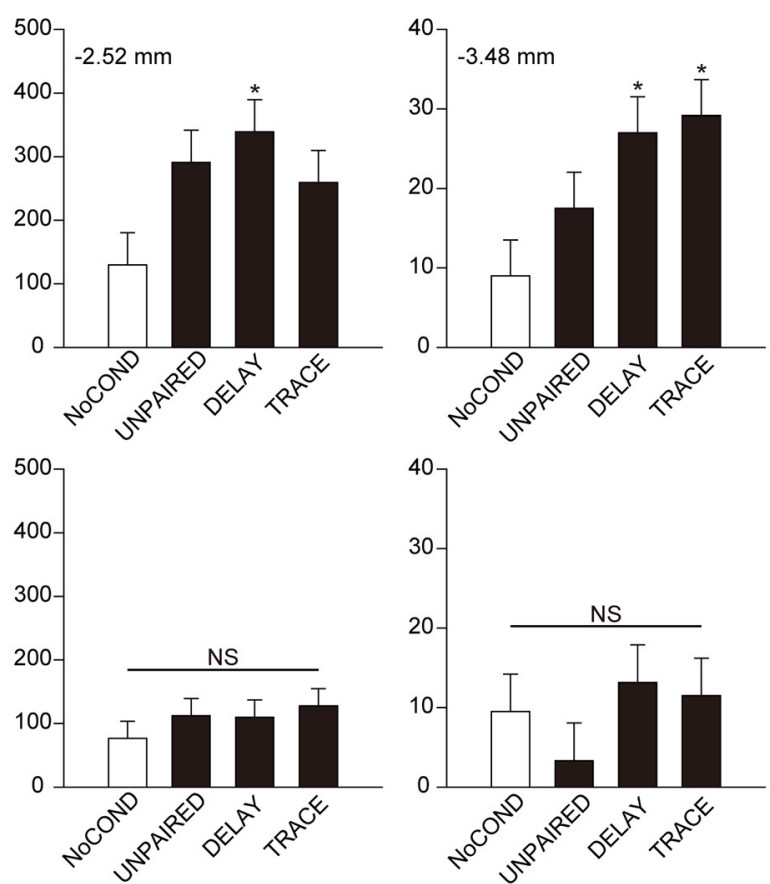

Figure 2. Re neurons were generally recruited in the encoding phase of trace and delay fear conditioning. $\boldsymbol{A}$, Animals underwent a 2-d behavioral experiment, in which half of the animals in each group were killed after conditioning and the rest after retrieval test. $\boldsymbol{B}, \mathrm{c}-\mathrm{Fos}+$ expression in Re neurons (red square). c-Fos signals were visualized with blue/black reaction products within the nucleus of activated neurons. $\boldsymbol{C}$, The freezing behavior of TRACE, DELAY, UNPAIRED, and NoCOND groups. Freezing levels of the conditioning ( $n=12$ per group) and test trials ( $n=6$ per group) are presented in left panel, respectively. The middle panel shows the averaged freezing levels of the UNPAIRED group (gray line with SEM) and the divergent high (solid lines) or low (dashed lines) freezing levels of each animal during retrieval test. The right panel shows the average c-Fos + count of the UNPAIRED group (gray bar with SEM) and the counts of high freezing (open circles; behaviors shown in solid lines) and low freezing (filled circles; behaviors shown in dashed lines) animals based on their behavior on trial 3 ( $n=3$ each). $\boldsymbol{D}$, Group differences in c-Fos + counts of the Re after conditioning (upper panel) and test (lower panel); $* p<0.05$. NS, not significant.

$\left.\eta_{\mathrm{p}}^{2}=0.71\right]$, and a significant interaction between group and trials $\left[F_{(9,60)}=6.33, p<0.001, \eta_{p}^{2}=0.49\right]$. Post hoc comparisons suggested that there was no statistical difference in freezing levels between the TRACE and DELAY groups $(p=0.80)$, which were both significantly higher than UNPAIRED or NoCOND group (all $p s<0.05$ ), indicating equivalent learning achieved by the two groups. The result was inconsistent with previous literature, i.e., generally lower freezing levels were observed following trace conditioning compared with delay conditioning 
(Raybuck and Lattal, 2014). Interestingly, the freezing level of the UNPAIRED group was significantly higher compared with NoCOND group $(p<0.05)$, yet significantly lower compared with DELAY and TRACE groups (both $p s<0.05)$. When we further looked into the freezing level of each animal in this group (Fig. $2 C$, middle panel), we found that the rats displayed two distinct behavioral patterns that half of them showed high fear (solid lines, $n=3$ ), whereas the other half showed low fear (dashed lines, $n=3$ ), to tones.

An example of $\mathrm{c}$-Fos expression in the Re is shown in Figure 2B. During the encoding phase (Fig. 2D, upper panel) of fear, ANOVA revealed significant main effects of group in all levels examined (relative to bregma, -1.56 , -2.52 , and $-3.48 \mathrm{~mm})\left[-1.56 \mathrm{~mm}, F_{(3,20)}=4.40, p=0.016\right.$, $\eta_{\mathrm{p}}^{2}=0.40 ;-2.52 \mathrm{~mm}, F_{(3,20)}=3.15, p=0.048, \eta_{\mathrm{p}}^{2}=0.32$; $-3.48 \mathrm{~mm}, F_{(3,20)}=4.20, p=0.019, \eta_{\mathrm{p}}^{2}=0.39$ ]. Post hoc comparisons suggested that compared with NoCOND group, the numbers of c-Fos + neurons were consistently higher in DELAY group in the $\operatorname{Re}($ all $p s<0.05$ ), while the cFos + counts of TRACE group were significantly higher at the level of $-3.48 \mathrm{~mm}(p<0.05)$. However, although cFos + counts also increased in the UNPAIRED group compared with NoCOND group, the differences did not reach significance in any of the levels we examined (all $p s>0.05$ ). During the retrieval phase (Fig. $2 D$, lower panel) of fear, ANOVA revealed a significant main effect of group $\left[F_{(3,20)}=3.68, p=0.029, \eta_{\mathrm{p}}^{2}=0.36\right]$ only at level $-1.56 \mathrm{~mm}$. Post hoc comparisons suggested that compared with NoCOND group, the significant difference is with the UNPAIRED group only $(p<0.05)$. When we further looked into the c-Fos activation level based on their freezing behavior in this group (Fig. 2C, middle panel), we found that the three animals with high freezing levels had low c-Fos activation (Fig. 2C, right panel, open circles; c-Fos count $=192,146$, and 95), consistent with those of DELAY and TRACE groups. On the contrary, the ones with low freezing levels were more likely to have high c-Fos activation (Fig. $2 C$, right panel, filled circles; c-Fos count $=438,299$, and $101)$ in the $\mathrm{Re}$, a result suggesting that this brain structure was recruited when the animals displayed no fear at the behavioral level. Together, these results revealed critical insights into the recruitment of the Re neurons during the encoding, but not the retrieval phase, of delay and trace fear memory.

\section{Experiment 2: ReRh is necessary for the acquisition phase of trace fear memory}

In experiment 1 , we demonstrated evidences that the $R e$ is recruited during the encoding of delay and trace fear memory. However, a recent study indicated that the ReRh is also important for the encoding of contextual fear (Ramanathan et al., 2018a). Thus, we cannot rule out the possibility that the neurons were activated because of contextual fear, rather than CS-induced fear. To address the question, we designed a series of behavioral pharmacology experiments (experiments 2-4) to determine whether the ReRh is necessarily recruited in delay and trace conditioning.

In this experiment, we examined the determinant role of the ReRh during the acquisition phase of trace fear (Fig.
$3 A)$. The placements of the injector tips for all the animals included in data analyses are summarized in Figure 3B. Of the initial 32 rats that underwent surgeries, one was excluded due to death during surgery and seven due to cannula misplacements, leading to the following final group sizes: V-TRACE $(n=7), \operatorname{M-TRACE}(n=6)$, V-DELAY $(n=6)$, and M-DELAY $(n=5)$.

On day 1 , the animals underwent the conditioning procedure (Fig. 3C, left panel). All animals showed an increase in freezing levels as the trials proceeded. There was a significant main effect of trials $\left[F_{(10,200)}=81.75\right.$, $\left.p<0.001, \eta_{\mathrm{p}}^{2}=0.80\right]$ and a significant two-way interaction between drug and trials $\left[F_{(10,200)}=2.22, p=0.018, \eta_{\mathrm{p}}^{2}\right.$ $=0.10]$. Under the influence of muscimol, these animals had slightly higher freezing levels compared with controls during $\mathrm{BL}$ and the first $\mathrm{CS}$. However, at the last trial of conditioning, there was no statistical difference in freezing levels among groups (all $p s>0.05$ ).

On day 2, all animals were tested for their acquisition of delay or trace conditioning under ReRh inactivation. (Fig. $3 C$, right panel). All animals demonstrated fear responses to the tones. There were significant main effects of group $\left[F_{(1,20)}=20.68, p<0.001, \eta_{\mathrm{p}}^{2}=0.51\right]$, drug $\left[F_{(1,20)}=15.30\right.$, $\left.p=0.001, \eta_{\mathrm{p}}^{2}=0.43\right]$, and trials $\left[F_{(10,200)}=18.01, p<0.001\right.$, $\left.\eta_{\mathrm{p}}^{2}=0.47\right]$. However, the lack of significant two-way interaction between group and drug $\left[F_{(1,20)}=1.34, p=0.26, \eta_{p}^{2}=\right.$ 0.06 ] suggested that both delay and trace procedures may have been affected by ReRh inactivation during acquisition. This result was inconsistent with the earlier literature, i.e., inactivation of the ReRh did not impair the acquisition of delay fear conditioning (Ramanathan et al., 2018a). We therefore did single-factor analysis for DELAY and TRACE groups to further examine the respective drug effects. For DELAY group, there was a significant main effect of trials $\left[F_{(10,90)}=24.61, p<0.001, \eta_{p}^{2}=0.73\right]$, but only a marginal effect of drug $\left[F_{(1,9)}=4.23, p=0.07, \eta_{p}^{2}=0.32\right]$. For TRACE group, there were significant main effects of drug $\left[F_{(1,11)}=12.29, p=0.005, \eta_{\mathrm{p}}^{2}=0.53\right]$ and trials $\left[F_{(10,110)}=\right.$ 4.76, $\left.p<0.001, \eta_{\mathrm{p}}^{2}=0.30\right]$. Together, our data suggested that ReRh inactivation during conditioning impaired fear encoding, and this effect was in majority attributed to the TRACE group.

\section{Experiment 3: ReRh is not necessary for the consolidation phase of trace fear memory}

In this experiment, we examined the role of the ReRh during the consolidation phase of trace fear (Fig. 4A). The placements of the injector tips for all the animals included in data analyses are summarized in Figure $4 B$. Of the initial 32 rats that underwent surgeries, one was excluded due to death during surgery and two due to cannula misplacements, leading to the following final group sizes: C-TRACE $(n=8)$, M-TRACE $(n=7)$, C-DELAY $(n=7)$, and M-DELAY $(n=7)$.

On day 1 , the animals underwent the conditioning procedure (Fig. 4C, left panel). All animals showed an increase in freezing levels as the trials proceeded. There was a significant main effect of trials $\left[F_{(10,250)}=51.77\right.$, $\left.p<0.001, \eta_{\mathrm{p}}^{2}=0.67\right]$ and a significant three-way interaction among group, drug, and trials $\left[F_{(10,250)}=2.09\right.$, $\left.p=0.026, \eta_{\mathrm{p}}^{2}=0.08\right]$. There were fluctuations in freezing 
A

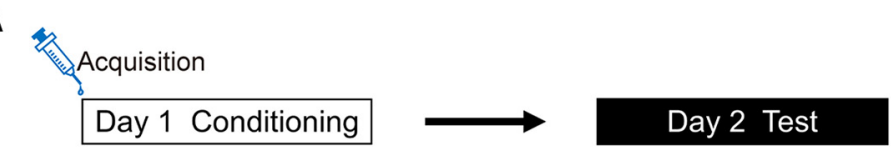

B
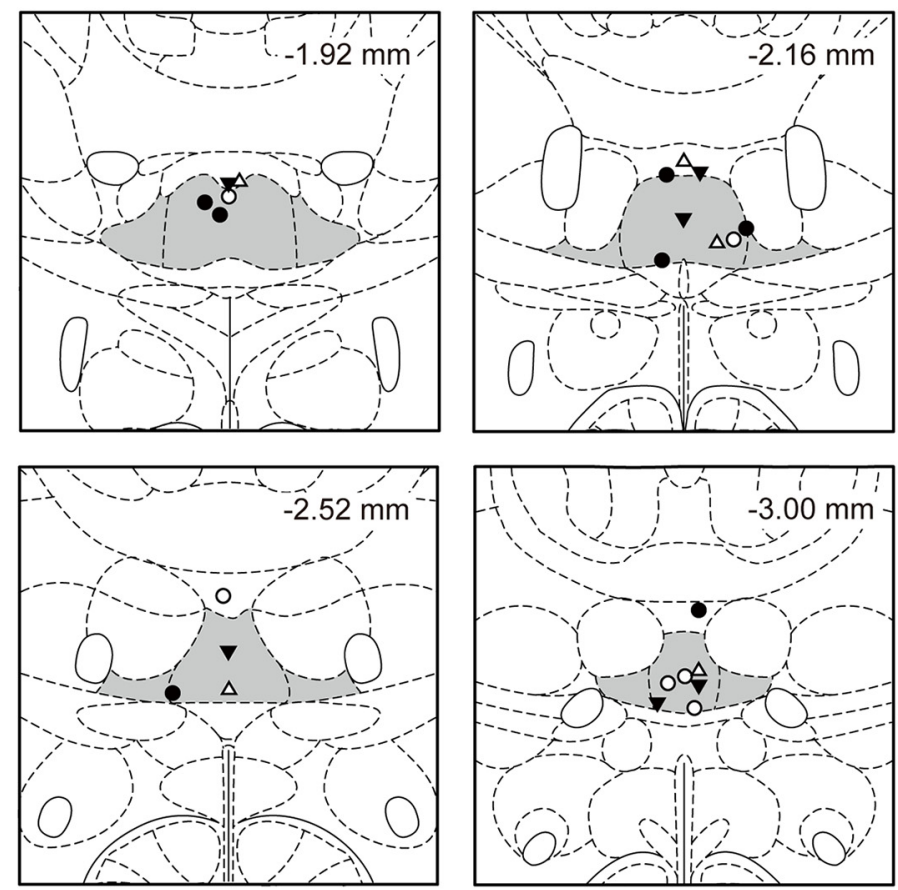

C

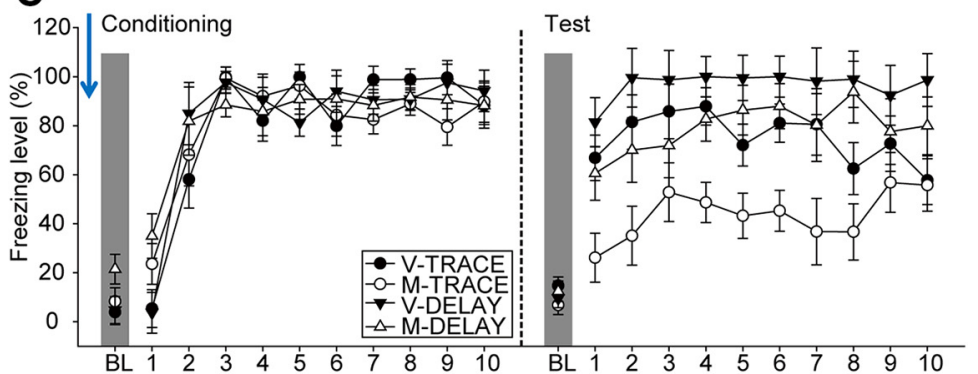

Figure 3. The ReRh was critically involved during the acquisition of trace fear conditioning. $\boldsymbol{A}$, Experimental design. Animals underwent a 2-d behavioral experiment, in which the animals received drug infusion immediately before conditioning (day 1 ). $\boldsymbol{B}$, Injector tip placements of all the animals included in data analyses at levels $-1.92,-2.16,-2.52$, and -3.00 mm posterior relative to bregma. The gray areas marked in the atlas are the Re and ventral Re. $\boldsymbol{C}$, The freezing behavior of V-TRACE $(n=7), \operatorname{M-TRACE}(n=6)$, V$\operatorname{DELAY}(n=6)$, and M-DELAY groups $(n=5)$ when injections were performed before conditioning.

levels among the four groups across trials. However, at the last trial of conditioning, there was no statistical difference in freezing levels among them (all $p s>0.05$ ). Because newly acquired memory is less stable and may be prone to any interference during consolidation (McGaugh, 2000), it is possible that potential behavioral effects may result from the infusion itself. To rule out this possibility, the controls were further divided into vehicle (DELAY, $n=4$; TRACE, $n=4$ ) and home (DELAY, $n=3$; TRACE, $n=4$ ). Animals in the vehicle group underwent vehicle infusion right after the conditioning procedure, whereas those in the home group went back to home cages after the conditioning procedure without further manipulation.

On day 2, all animals were tested for their consolidation of delay or trace conditioning under ReRh inactivation. We first double-checked the behavioral performances between the vehicle and the home groups. Because there were no statistical differences in any of the main effect or interactions regarding postconditioning vehicle infusion during retrieval test (all $p s \geq 0.05$ ), the two groups were merged as respective control groups (C-TRACE and CDELAY) in the following analyses. All animals showed high levels of CS-elicited freezing behavior after tone onset and slightly decreased toward later trials (Fig. $4 C$, right panel). There were significant main effects of group $\left[F_{(1,25)}=7.74\right.$, $\left.p=0.01, \eta_{\mathrm{p}}^{2}=0.24\right]$ and trials $\left[F_{(10,250)}=27.12, p<0.001\right.$, $\left.\eta_{\mathrm{p}}^{2}=0.52\right]$, and a significant two-way interaction between group and trials $\left[F_{(10,250)}=2.67, p=0.004, \eta_{\mathrm{p}}^{2}=0.10\right]$. However, there were no statistical differences in the main effect of drug $\left[F_{(1,25)}<1, \eta_{\mathrm{p}}^{2}=0.01\right]$, two-way interactions between drug and group $\left[F_{(1,25)}<1, \eta_{\mathrm{p}}^{2}=0.03\right]$ or drug and trials $\left[F_{(10,250)}=1.30, p=0.23, \eta_{p}^{2}=0.05\right]$, or three-way 
A

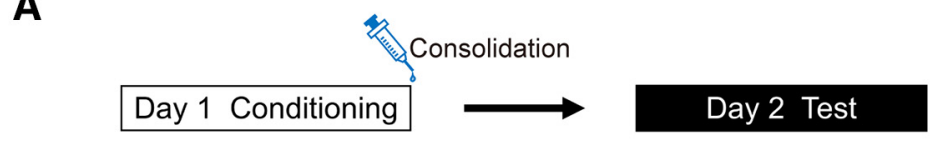

B
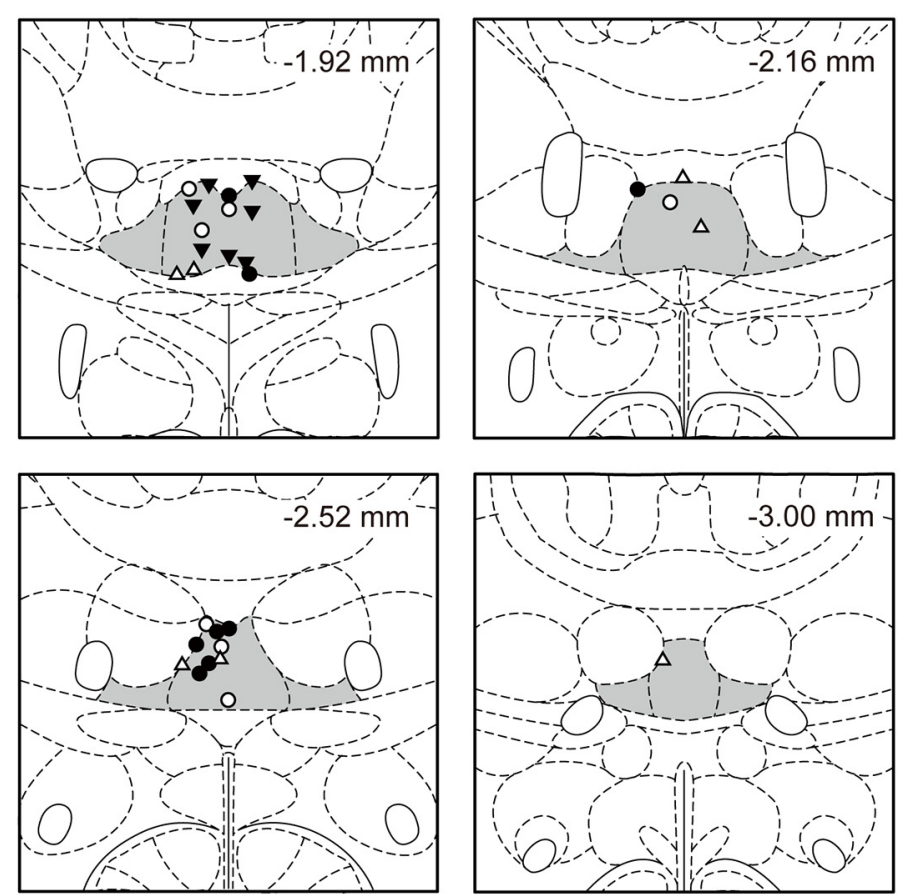

C

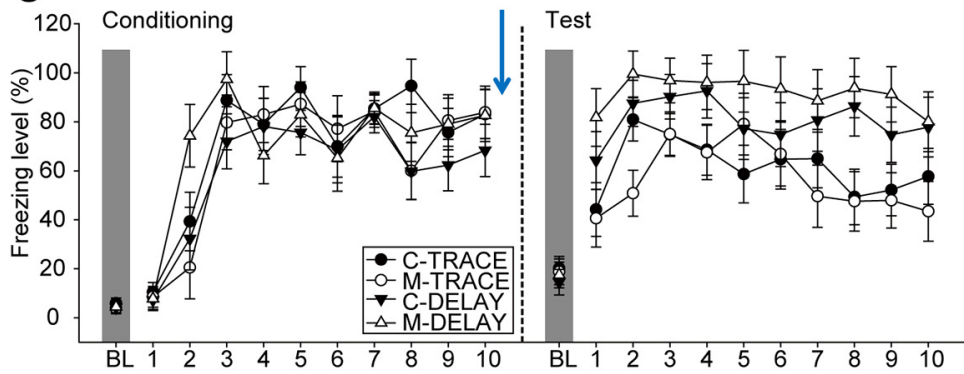

Figure 4. The ReRh was not involved during the consolidation of trace fear conditioning. A, Experimental design. Animals underwent a 2-d behavioral experiment, in which the animals received drug infusion immediately after conditioning (day 1 ). $\boldsymbol{B}$, Injector tip placements of all the animals included in data analyses at levels $-1.92,-2.16,-2.52$, and -3.00 mm posterior relative to bregma. The gray areas marked in the atlas are the Re and ventral Re. $\boldsymbol{C}$, The freezing behavior of C-TRACE $(n=8)$, M-TRACE $(n=7)$, CDELAY $(n=7)$, and M-DELAY groups $(n=7)$ when injections were performed after conditioning.

interaction among drug, group, and trials $\left[F_{(10,250)}<1, \eta_{\mathrm{p}}^{2}=\right.$ $0.03]$. The lack of drug main effect and its interactions suggested that postconditioning inactivation of the ReRh did not affect the consolidation of trace fear memory. The higher freezing levels in DELAY group compared with TRACE group and the faster decline in freezing levels toward later trials in TRACE group supported the idea that the CS-US association is weaker in trace fear conditioning (Raybuck and Lattal, 2014).

\section{Experiment 4: ReRh is not necessary for the retrieval phase of trace fear memory}

In this experiment, we examined the role of the ReRh during the retrieval phase of trace fear (Fig. 5A). The placements of the injector tips for all the animals included in data analyses are summarized in Figure $5 B$. Of the initial 32 rats that underwent surgeries, one was excluded due to death during surgery and five due to cannula misplacements, leading to the following final group sizes: $V$ TRACE $(n=5)$, M-TRACE $(n=7)$, V-DELAY $(n=7)$, and M$\operatorname{DELAY}(n=7)$.

On day 1 , the animals underwent the conditioning procedure (Fig. 5C, left panel). All animals showed an increase in freezing levels as the trials proceeded. There was a significant main effect of trials $\left[F_{(10,220)}=49.85\right.$, $\left.p<0.001, \eta_{\mathrm{p}}^{2}=0.70\right]$ and a significant two-way interaction between group and trials $\left[F_{(10,220)}=2.30, p=0.014\right.$, $\left.\eta_{\mathrm{p}}^{2}=0.10\right]$. Although there were fluctuations in freezing levels between delay or trace procedures across trials, there was no statistical difference in freezing levels 
A

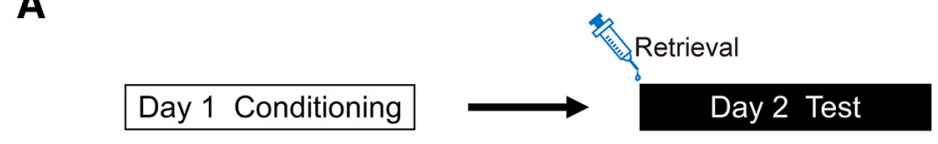

B
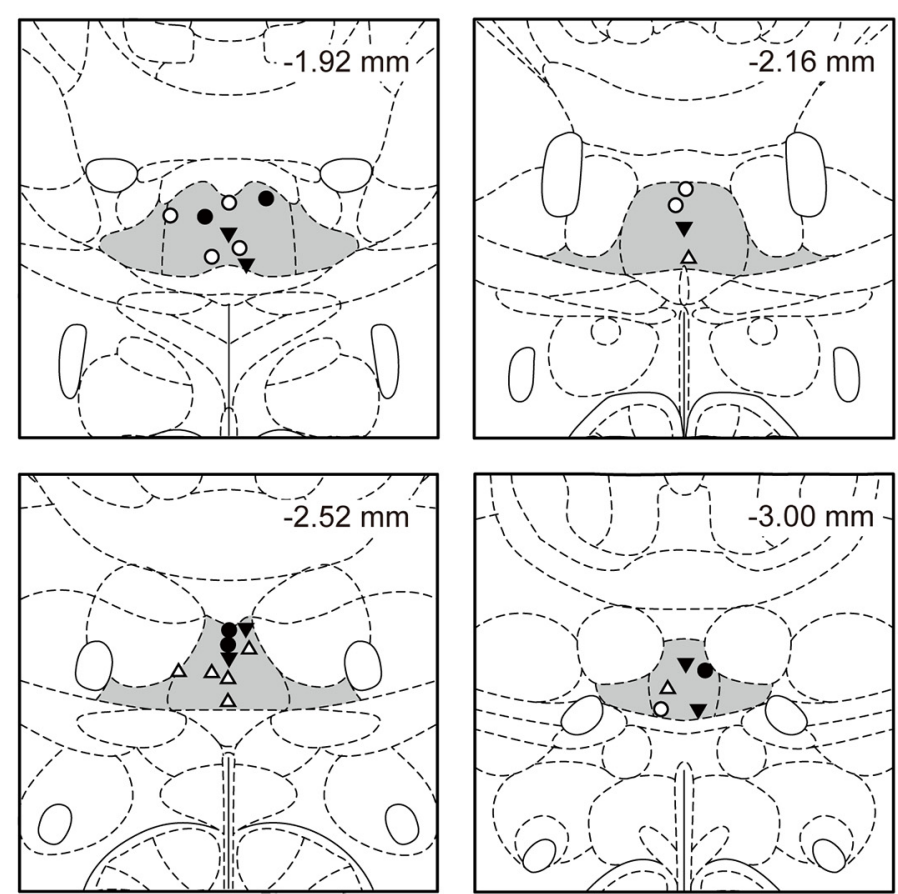

C

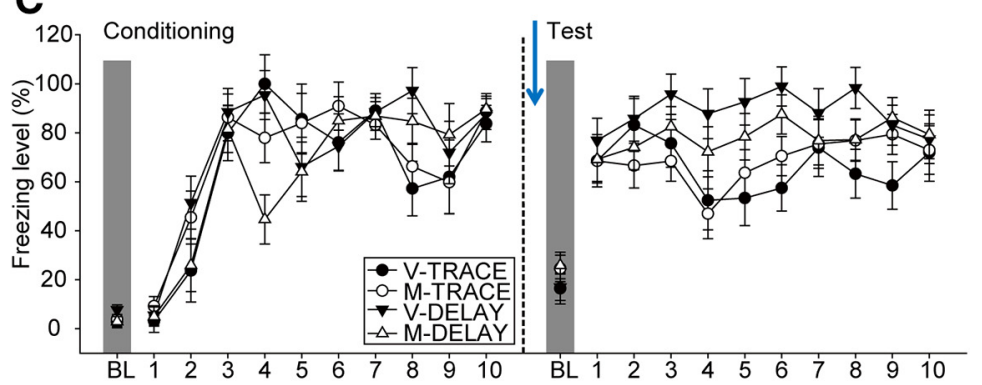

Figure 5. The ReRh was not involved during the retrieval of trace fear conditioning. $\boldsymbol{A}$, Experimental design. Animals underwent a 2$\mathrm{d}$ behavioral experiment, in which the animals received drug infusion immediately before test (day 2). $\boldsymbol{B}$, Injector tip placements of all the animals included in data analyses at levels $-1.92,-2.16,-2.52$, and $-3.00 \mathrm{~mm}$ posterior relative to bregma. The gray areas marked in the atlas are the Re and ventral Re. $\boldsymbol{C}$, The freezing behavior of V-TRACE $(n=5), \operatorname{M-TRACE}(n=7)$, V-DELAY $(n=7)$, and M-DELAY groups $(n=7)$ when injections were performed before retrieval test.

among groups at the last trial of conditioning (all ps $>0.05)$.

On day 2, all animals were tested for their retrieval of delay or trace conditioning under ReRh inactivation. All animals showed high levels of CS-elicited freezing behavior after tone onset and the freezing levels maintained high throughout the test session (Fig. 5C, right panel). There were significant main effects of group $\left[F_{(1,22)}=5.36\right.$, $\left.p=0.03, \eta_{\mathrm{p}}^{2}=0.20\right]$ and trials $\left[F_{(10,220)}=23.21, p<0.001\right.$, $\left.\eta_{\mathrm{p}}^{2}=0.51\right]$, and a significant two-way interaction between group and trials $\left[F_{(10,22)}=2.27, p=0.015, \eta_{\mathrm{p}}^{2}=0.10\right]$. However, there were no statistical differences in the main effect of drug $\left[F_{(1,22)}<1, \eta_{\mathrm{p}}^{2}=0.01\right]$, two-way interactions between drug and group $\left[F_{(1,22)}<1, \eta_{\mathrm{p}}^{2}=\right.$ $0.04]$ or drug and trials $\left[F_{(10,220)}=1.25, p=0.26, \eta_{\mathrm{p}}^{2}=\right.$
0.05], or three-way interaction among drug, group, and trials $\left[F_{(10,220)}<1, \eta_{\mathrm{p}}^{2}=0.03\right]$. The lack of drug main effect and its interactions suggested that pre-test inactivation of the ReRh did not affect the retrieval of trace fear memory. The higher freezing levels in DELAY group compared with TRACE group was consistent with the results of experiment 3 showing that CS-US association was weaker in trace fear conditioning.

To sum up experiments $2-4$, our results revealed that inactivation of the ReRh impaired the acquisition, but not the consolidation or retrieval, of trace fear. This finding is in line with the results in experiment 1, further supporting its critical role in the encoding of trace fear. However, pharmacological inactivation of the ReRh at any of the phases we examined did not interfere with delay fear conditioning. 
A

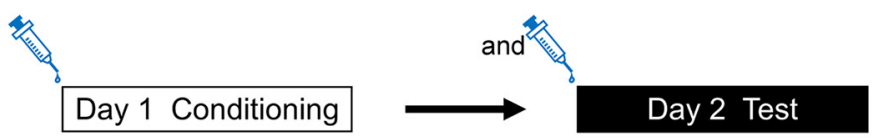

B
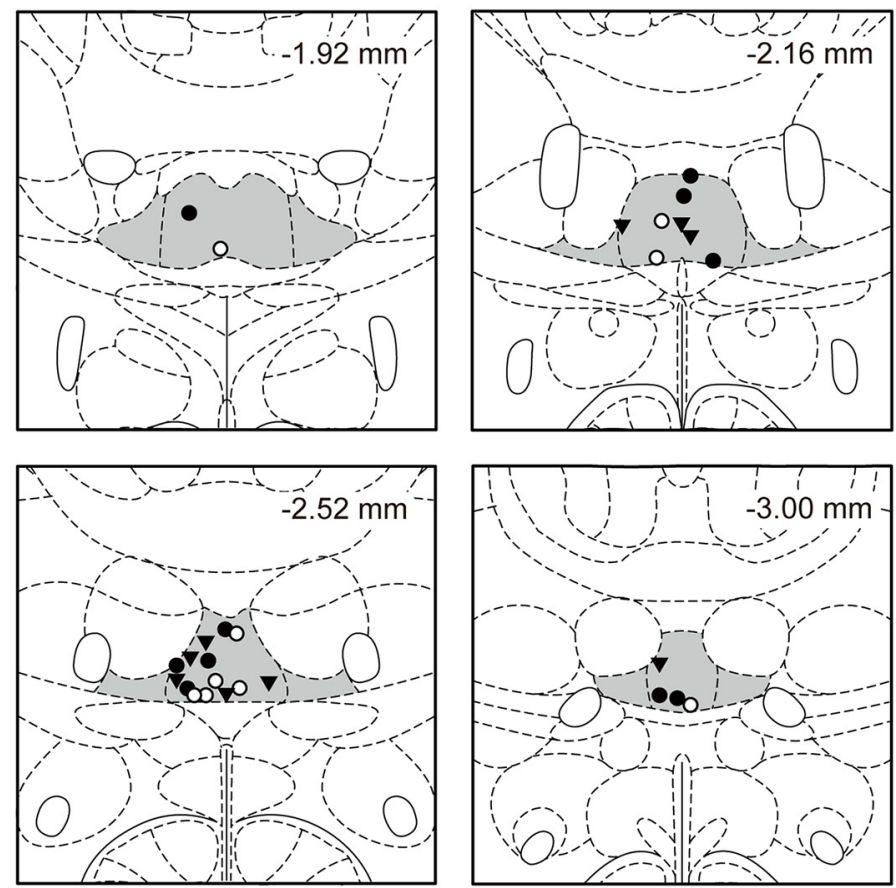

C

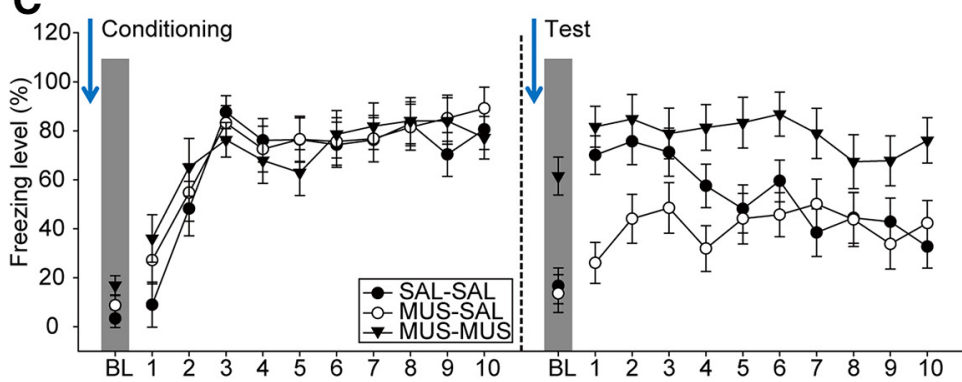

Figure 6. Retrieval of trace fear acquired under ReRh inactivation reprised under ReRh inactivation. $\boldsymbol{A}$, Experimental design. Animals underwent a 2-d behavioral experiment, in which the animals received drug infusion immediately before both conditioning and retrieval test. $\boldsymbol{B}$, Injector tip placements of all the animals included in data analyses at levels $-1.92,-2.16,-2.52$, and -3.00 $\mathrm{mm}$ posterior relative to bregma. The gray areas marked in the atlas are the Re and ventral Re. $\boldsymbol{C}$, The freezing behavior of SAL-SAL $(n=10)$, MUS-SAL $(n=9)$, and MUS-MUS groups $(n=9)$ when the injections were performed before both conditioning and retrieval test.

\section{Experiment 5: retrieval of trace fear acquired under ReRh inactivation reprised under ReRh inactivation}

In experiment 2, we established that the ReRh is critically involved in the acquisition of trace fear. However, earlier literature suggested that retrieval of contextual fear acquired under ReRh inactivation is state dependent (Ramanathan et al., 2018a). To examine whether this is also true for trace fear, we brought the ReRh off-line with muscimol throughout the entire process (Fig. 6A). We reasoned that if the trace fear memory acquired under ReRh inactivation is indeed state dependent, then fear to the tones would reprise under ReRh inactivation during retrieval test in this experiment. As our results in experiment 4 indicated that the ReRh is not necessary for the retrieval phase of trace fear memory, SAL-MUS group was not included. The placements of the injector tips for all the animals included in data analyses are summarized in Figure $6 B$. Of the initial 36 rats that underwent surgeries, two were excluded due to death during surgery and six due to cannula misplacements, leading to the following final group sizes: SAL-SAL $(n=10)$, MUS-SAL $(n=9)$, and MUS-MUS $(n=9)$.

On day 1, all animals showed an increase in freezing levels toward later trials during trace conditioning regardless of the injections (Fig. 6C, left panel). This is consistent with our results in experiment 2 , indicating that ReRh inactivation did not interfere with fear expression. There was a significant main effect of trials $\left[F_{(10,250)}=46.15\right.$, 
$\left.p<0.001, \eta_{\mathrm{p}}^{2}=0.65\right]$. However, there were no significant main effect of group $\left[F_{(2,25)}<1, \eta_{\mathrm{p}}^{2}=0.01\right]$ or two-way interaction between group and trials $\left[F_{(20,250)}=1.17\right.$, $\left.p=0.28, \eta_{p}^{2}=0.09\right]$. All groups reached equivalent high levels of freezing at the last trial (all $p s>0.05$ ).

On day 2, we replicated our findings in experiment 2 that compared with SAL-SAL group, MUS-SAL animals demonstrated impaired acquisition of trace fear. Importantly, freezing levels of MUS-MUS group remained high throughout the test session from $\mathrm{BL}$ to the last trial (Fig. 6C, right panel). There were significant main effects of group $\left[F_{(2,25)}=8.53, p=0.001, \eta_{\mathrm{p}}^{2}=0.41\right]$ and trials $\left[F_{(10,250)}=\right.$ $\left.6.46, p<0.001, \eta_{\mathrm{p}}^{2}=0.21\right]$, and a significant interaction between group and trials $\left[F_{(20,250)}=1.93, p=0.011, \eta_{p}^{2}=\right.$ $0.13]$. Compared with SAL-SAL group, post hoc comparisons suggested that freezing levels of MUS-SAL group were significantly lower in trial $1(p<0.05)$ and marginally lower in trial $2(p=0.076)$, whereas freezing levels of MUSMUS group were significantly higher in BL, trial 7 , and trial 10 (all $p s<0.05$ ). Together, our results suggested that there was a state-dependent retrieval of trace memory that fear acquired under ReRh inactivation only reprised under ReRh inactivation. However, the high levels of BL freezing in the MUS-MUS group and its slower decline in amplitude toward later trials compared with SAL-SAL group also suggested that the fear was generalized.

\section{Discussion}

In the c-Fos visualization experiment, across the antero-posterior axis we examined, the Re was generally recruited in the encoding of delay fear conditioning, whereas the posterior Re was recruited in the encoding of trace fear conditioning. Pharmacological inactivation of the ReRh at different time points further illustrated that the ReRh was crucially involved in the acquisition phase specifically of trace fear. Additionally, trace memory acquired under ReRh inactivation only reprised during ReRh inactivation. In conclusion, our results uncovered a critical role of the ReRh in trace learning at the behavioral level.

A growing body of anatomic, physiological, and behavioral literature has been conducted on the role of the $\mathrm{ReRh}$ in behavior and cognition (Cassel et al., 2013; Dolleman-van der Weel et al., 2019). ReRh lesions impaired the spatial representation encoded by hippocampal CA1 place cells by reducing the place field stability and firing variability, indicating the involvement of the ReRh in spatial cognition (Cholvin et al., 2018). Others reported that the ReRh is recruited in navigation by participating in the neurocircuitry involved in the representation of goal-directed routes and mediating the intended movement of the animal (Ito et al., 2015, 2018). Additionally, the ReRh is critical for fear extinction (Ramanathan et al., 2018b), a procedure that involves the HPC in the encoding phase and context-dependent expression of fear after extinction (Maren et al., 2013). Overall, these studies unveil the indispensable character the ReRh plays in hippocampal-dependent memory and tasks. Moreover, the ReRh is involved in cognitive functions that recruit the mPFC-HPC circuitry, such as spatial memory (Loureiro et al., 2012; Mei et al., 2018; Klein et al., 2019), spatial working memory (Layfield et al., 2015; Viena et al., 2018), recognition memory (Barker and Warburton, 2018), sequential memory (Jayachandran et al., 2019), and contextual fear memory (Ramanathan et al., 2018a; Quet et al., 2020).

Despite the abundance of research investigating the role of the ReRh on different demands, studies regarding the tasks that rely on association of stimuli with temporal discontinuity in training, such as trace conditioning, were sparse. Previous studies using pharmacological approaches indicated that trace fear learning was impaired by pre-conditioning unilateral (Gilmartin et al., 2012) or bilateral inactivation of the HPC (Esclassan et al., 2009), as well as bilateral inactivation of the mPFC (Gilmartin and Helmstetter, 2010). Based on these findings, it is speculated that the ReRh serves as a critical node in the information flow from the MPFC to the HPC in trace fear conditioning. Indeed, here we demonstrated that the ReRh is specifically involved in the acquisition phase of trace fear conditioning, supporting its vital role in the encoding of CS-US temporal relationship.

The participation of the ReRh during the acquisition phase of behavioral tasks has been repeatedly demonstrated. For example, inactivation of the ReRh impaired the acquisition of contextual conditioning (Ramanathan et al., 2018a). A recent study using lesion and pharmacology methods revealed the importance of the ReRh in the establishment of depression and its underlying neuromorphological and endocrine effects (Kafetzopoulos et al., 2018). Additionally, optogenetic inhibition during the sample phase, but not the delay or choice phase, significantly decreased choice accuracy in spatial working memory task (Maisson et al., 2018). These earlier results, together with our current findings, point to the convergent recruitment of the mPFC-HPC circuitry in cognitive tasks and emotional processing, in which the ReRh is important in mediating the information exchange during the initial encoding (Hoover and Vertes, 2012). On the contrary, the results are inconsistent regarding its role during the retrieval of memory. For example, ReRh inactivation impaired spatial working memory performance in a delay-dependent manner (Layfield et al., 2015). Using an hM4Di synaptic-silencing approach, it has been shown that the mPFC-Re projection regulates sequential memory retrieval (Jayachandran et al., 2019). On the other hand, the ReRh is not involved in the retrieval process of recent spatial memory (Loureiro et al., 2012) and contextual memory (Ramanathan et al., 2018a). In our study, there were no behavioral effects of ReRh inactivation on consolidation (experiment 3) or retrieval (experiment 4) of trace fear. However, because ten CS-US trials were administered during conditioning in our series of experiments, in which the freezing levels reached a plateau after trial 3, we cannot rule out the possibility that the lack of behavioral effects was due to over-training. It is also worth noticing that in rats, memories become resilient to interferences and stabilized $6 \mathrm{~h}$ after training (McGaugh, 1966). The "early phase" of memory consolidation corresponds to synaptic consolidation, which is mediated by intracellular molecular mechanisms and involves changes in 
the synaptic connections. On the other hand, the "late phase" consolidation correspond to systems consolidation, which requires the reorganization of the memory trace throughout systems level (Dudai, 2004). Immediate postconditioning inactivation of the ReRh (experiment 3) interfered with only the early phase of memory consolidation, which is within hours when long-term potentiation (LTP) is independent of protein synthesis (McGaugh, 2000). The effect of ReRh inactivation on trace fear conditioning during late phase consolidation, which lasts several days and requires gene expression (Abel and Lattal, 2001; Lin et al., 2003) and protein synthesis (Schafe et al., 2000; Santini et al., 2004), is beyond the scope of the present study. Notably, Sierra et al., recently showed that lidocaine-induced inhibition of the Re inhibited LTP induction in the CA1-anterior cingulate cortex pathways (Sierra et al., 2017). Indeed, ReRh lesion impaired remote contextual memory (Quet et al., 2020) and spatial memory (Loureiro et al., 2012; Klein et al., 2019). Together, these studies provide evidence of the role of the ReRh in mediating systems consolidation and memory persistence.

In experiment 1, we showed that the freezing levels between the TRACE and DELAY groups were equivalent during test (Fig. 2C, left panel). This behavioral result was inconsistent with previous literature, i.e., generally lower freezing levels following trace conditioning compared with delay conditioning (Raybuck and Lattal, 2014). Different from experiments 2-5, only three CSs were given in this experiment on day 2 because the number of tones presented would initiate different processes. Brief re-exposure of the CS tends to engage memory retrieval followed by reconsolidation, while prolonged re-exposure engages extinction (Merlo et al., 2014; Cahill and Milton, 2019). Thus, the brief re-exposure in experiment 1 ensured that c-Fos expression corresponded to fear retrieval, but not fear extinction (Bouton, 2004). The weaker CS-US association by trace procedure may require more trials to emerge. Indeed, when $10 \mathrm{CSs}$ were presented during the retrieval tests (experiments 3 and 4), DELAY groups showed stronger CS-US associations compared with TRACE groups (significant main effect of group), and there was a faster decline of freezing levels in TRACE groups (significant interactions between group and trials).

During the encoding phase in experiment 1 (Fig. 2D, upper panel), the UNPAIRED group showed up-shifts in the c-Fos expressions compared with NoCOND group. Several factors may have contributed to the result. For example, earlier studies have shown that encoding of contextual fear is ReRh dependent (Ramanathan et al., 2018a). Moreover, increased c-Fos expression was observed in the Re when rats were subjected to peripheral noxious stimulation (Bullitt, 1990). However, we did not have the animals that underwent "contextual fear conditioning procedure" (i.e., no tones during conditioning) or "shock-only procedure" (i.e., animals received footshocks in another novel context) in this study. Without these two critical control groups, we cannot rule out the possibility that the increase in c-Fos expression was a consequence of contextual fear encoding or a consequence of footshocks. Nonetheless, such increase in c-Fos expression in the UNPAIRED group did not reach significance in any of the coronal levels we examined (all $p s>0.05$ ). This result suggested a pivotal role of the CS-US temporal contingency in recruiting the Re during encoding of fear memory. We also acknowledged the limitation that there were no other control brain regions reported in this study. Thus, we could not conclude whether there was a general up-shift in c-Fos expression at the circuitry level.

Many past studies considered the unpaired procedures as behavioral controls to demonstrate that animals would not acquire CS-US associations under such training procedure (Gormezano et al., 1982; Papini and Bitterman, 1990; Burman et al., 2014). Interestingly, our data showed that the UNPAIRED animals demonstrated divergent high or low freezing levels during tone test (Fig. 2C). What CSUS relationship the animals acquired, despite that the CSs did not explicitly predict the occurrence of USs in unpaired procedures, awaits further identification. We suspected that the UNPAIRED animals in our study may have adopted different strategies to cope with an upcoming threat, the US. Some animals may have still acquired the CS-US association despite the long trace intervals, and thus demonstrated high freezing levels during CSs. Since the Re was not required during retrieval after the animals have acquired the trace fear, low c-Fos expression was observed, in line with the DELAY and TRACE groups. On the other hand, some animals may have instead considered the CSs as safety signals, in that with such long trace intervals, the CSs signal the absence of the USs for a certain period of time (Moscovitch and LoLordo, 1968; Kalat and Rozin, 1973). Under such scenario, the animals showed low fear during the CSs at the behavioral level. However, the relatively high c-Fos expression indicated the recruitment of the $\mathrm{Re}$ neurons at the neurobiological level. Indeed, earlier studies have shown that theta activity in the HPC differed when the animals were exposed to danger and safety signals (Kasicki et al., 2009) and that the circuit of mPFC and basolateral amygdala was dynamically engaged in fear discrimination between CS(safety signal) and CS+ (Likhtik et al., 2014). The Re, which has reciprocal projections with the HPC and the $\mathrm{mPFC}$, is therefore likely to participate in the coordination of the neurocircuitry of safety signals. It is worth noticing that the context and the CS always compete for associative strength with the US (Phillips and LeDoux, 1994; Marchand et al., 2014). Phillips and LeDoux suggested that in the procedures with paired CS-US associations, the phasic tone CS is the primary stimulus that associates with the US with contextual cues occurring in the background. On the contrary, when there is no phasic CS that is explicitly paired with a US, i.e., unpaired procedure, the primary associations are between the US and static contextual stimuli, which are therefore in the foreground (Phillips and LeDoux, 1994). Their results, together with Marchand et al. (2014)'s findings, demonstrated that animals in the unpaired group froze less during the CS test and more during context test when compared with paired groups (Phillips and LeDoux, 1994; Marchand et al., 2014). However, in this current study, we did not have an independent context test back to the original conditioning context to examine such scenario. 
A recent study suggested that the $R e R h$ is essential for hippocampal-dependent encoding of "precise" contextual memories and that the memory deficit induced by ReRh inactivation could be rescued when the ReRh was also offline during retrieval test (Ramanathan et al., 2018a). The authors proposed that animals without a functional ReRh could acquire an "elemental" representation of the context and associate it with the USs using a non-hippocampal system. Associations based on only one or few salient features of the behavioral procedure may result in difficulty to discriminate different contexts, and therefore more likely to generalize fear outside the conditioning context. Since the ReRh serves as a critical node between the hippocampal system and the mPFC to support trace fear learning, we examined whether bringing the ReRh off-line during the entire process of trace procedure may also lead to reprised trace fear during retrieval. Indeed, freezing levels of these animals remained high throughout the test session from BL to the last trial (MUS-MUS group; Fig. 6C, right panel). The results are likely a combined effect of generalized fear to context and tones without a functional ReRh during the trace procedure. High freezing levels during BL were consistent with earlier studies (Xu and Südhof, 2013; Ramanathan et al., 2018a) that the animals were less capable to differentiate contexts and showed fear generalization to a novel context. Moreover, these animals were also slower in the decline of their freezing levels to the tones compared with controls at the later trials. Different from the V-TRACE group in experiment 2, the SAL-SAL group expressed faster decline in freezing levels. This result could be attributed to different cohorts of animals being used, and to the non-specific effect caused by our manipulation, i.e., two infusions in experiment 5. Additional experiments to dissociate the relative contribution of fear generalization to contexts versus trace fear to tones are needed. Nonetheless, the ReRh serving as a node of the mPFCHPC circuitry may be critical in both precise encodings of contextual and temporal associations.

In summary, the findings that the ReRh serves as a central character in encoding the trace fear may provide crucial insights into the research regarding fear-related mental illnesses. Trace fear conditioning serves as a suitable model to conceive human emotional learning as human fear learning sometimes involves a trace temporal interval. Moreover, because this procedure recruits higher order nervous system (Bangasser et al., 2006; Gilmartin and Helmstetter, 2010), trace fear conditioning is also used to study cognitive functions. In recent decades, trace conditioning has been adapted to screen for cognitive disruption in mouse disease models for schizophrenia (Brzózka and Rossner, 2013) and Alzheimer's disease (Kaczorowski et al., 2011). Regarding the multiple roles of the ReRh in fear conditioning, depression, and stress-induced behaviors (Kafetzopoulos et al., 2018), research looking into the ReRh and underlying neurocircuitry of trace fear conditioning will provide a further understanding of fear behavior and mental disorders.

\section{References}

Abel T, Lattal KM (2001) Molecular mechanisms of memory acquisition, consolidation and retrieval. Curr Opin Neurobiol 11:180-187.
Agster KL, Burwell RD (2009) Cortical efferents of the perirhinal, postrhinal, and entorhinal cortices of the rat. Hippocampus 19:1159-1186.

Bangasser DA, Waxler DE, Santollo J, Shors TJ (2006) Trace conditioning and the hippocampus: the importance of contiguity. J Neurosci 26:8702-8706.

Barker GRI, Warburton EC (2018) A critical role for the nucleus reuniens in long-term, but not short-term associative recognition memory formation. J Neurosci 38:3208-3217.

Beylin AV, Gandhi CC, Wood GE, Talk AC, Matzel LD, Shors TJ (2001) The role of the hippocampus in trace conditioning: temporal discontinuity or task difficulty? Neurobiol Learn Mem 76:447-461.

Blum S, Hebert AE, Dash PK (2006) A role for the prefrontal cortex in recall of recent and remote memories. Neuroreport 17:341-344.

Bouton ME (2004) Context and behavioral processes in extinction. Learn Mem 11:485-494.

Brzózka MM, Rossner MJ (2013) Deficits in trace fear memory in a mouse model of the schizophrenia risk gene TCF4. Behav Brain Res 237:348-356.

Bullitt E (1990) Expression of c-fos-like protein as a marker for neuronal activity following noxious stimulation in the rat. J Comp Neurol 296:517-530.

Burman MA, Simmons CA, Hughes M, Lei L (2014) Developing and validating trace fear conditioning protocols in C57BL/6 mice. J Neurosci Methods 222:111-117.

Burwell RD, Amaral DG (1998) Cortical afferents of the perirhinal, postrhinal, and entorhinal cortices of the rat. J Comp Neurol 398:179-205.

Cahill EN, Milton AL (2019) Neurochemical and molecular mechanisms underlying the retrieval-extinction effect. Psychopharmacology (Berl) 236:111-132.

Cassel JC, Pereira de Vasconcelos A, Loureiro M, Cholvin T, Dalrymple-Alford JC, Vertes RP (2013) The reuniens and rhomboid nuclei: neuroanatomy, electrophysiological characteristics and behavioral implications. Prog Neurobiol 111:34-52.

Cholvin T, Hok V, Giorgi L, Chaillan FA, Poucet B (2018) Ventral midline thalamus is necessary for hippocampal place field stability and cell firing modulation. J Neurosci 38:158-172.

Delatour B, Witter MP (2002) Projections from the parahippocampal region to the prefrontal cortex in the rat: evidence of multiple pathways. Eur J Neurosci 15:1400-1407.

Dolleman-van der Weel MJ, Griffin AL, Ito HT, Shapiro ML, Witter MP, Vertes RP, Allen TA (2019) The nucleus reuniens of the thalamus sits at the nexus of a hippocampus and medial prefrontal cortex circuit enabling memory and behavior. Learn Mem 26:191205.

Dudai Y (2004) The neurobiology of consolidations, or, how stable is the engram? Annu Rev Psychol 55:51-86.

Esclassan F, Coutureau E, Di Scala G, Marchand AR (2009) Differential contribution of dorsal and ventral hippocampus to trace and delay fear conditioning. Hippocampus 19:33-44.

Fritz CO, Morris PE, Richler JJ (2012) Effect size estimates: current use, calculations, and interpretation. J Exp Psychol Gen 141:2-18.

Gilmartin MR, Helmstetter FJ (2010) Trace and contextual fear conditioning require neural activity and NMDA receptor-dependent transmission in the medial prefrontal cortex. Learn Mem 17:289296.

Gilmartin MR, Kwapis JL, Helmstetter FJ (2012) Trace and contextual fear conditioning are impaired following unilateral microinjection of muscimol in the ventral hippocampus or amygdala, but not the medial prefrontal cortex. Neurobiol Learn Mem 97:452-464.

Gilmartin MR, Miyawaki H, Helmstetter FJ, Diba K (2013) Prefrontal activity links nonoverlapping events in memory. J Neurosci 33:10910-10914.

Gormezano I, Kehoe E, Marshall BS (1982) Twenty years of classical conditioning with the rabbit. Prog Psychobiol Physiol Psychol 10:269-275.

Hallock HL, Wang A, Shaw CL, Griffin AL (2013) Transient inactivation of the thalamic nucleus reuniens and rhomboid nucleus 
produces deficits of a working-memory dependent tactile-visual conditional discrimination task. Behav Neurosci 127:860-866.

Hallock HL, Wang A, Griffin AL (2016) Ventral midline thalamus is critical for hippocampal-prefrontal synchrony and spatial working memory. J Neurosci 36:8372-8389.

Hoover WB, Vertes RP (2012) Collateral projections from nucleus reuniens of thalamus to hippocampus and medial prefrontal cortex in the rat: a single and double retrograde fluorescent labeling study. Brain Struct Funct 217:191-209.

Ito HT, Zhang SJ, Witter MP, Moser El, Moser MB (2015) A prefrontal-thalamo-hippocampal circuit for goal-directed spatial navigation. Nature 522:50-55.

Ito HT, Moser El, Moser MB (2018) Supramammillary nucleus modulates spike-time coordination in the prefrontal-thalamo-hippocampal circuit during navigation. Neuron 99:576-587.e5.

Jayachandran M, Linley SB, Schlecht M, Mahler SV, Vertes RP, Allen TA (2019) Prefrontal pathways provide top-down control of memory for sequences of events. Cell Rep 28:640-654.e6.

Kaczorowski CC, Sametsky E, Shah S, Vassar R, Disterhoft JF (2011) Mechanisms underlying basal and learning-related intrinsic excitability in a mouse model of Alzheimer's disease. Neurobiol Aging 32:1452-1465.

Kafetzopoulos V, Kokras N, Sotiropoulos I, Oliveira JF, LeiteAlmeida H, Vasalou A, Sardinha VM, Papadopoulou-Daifoti Z, Almeida OFX, Antoniou K, Sousa N, Dalla C (2018) The nucleus reuniens: a key node in the neurocircuitry of stress and depression. Mol Psychiatry 23:579-586.

Kalat JW, Rozin P (1973) Learned safety" as a mechanism in longdelay taste-aversion learning in rats. J Comp Physiol Psychol 83:198-207.

Kasicki S, Jeleń P, Olszewski M, Sławińska U (2009) Electrical hippocampal activity during danger and safety signals in classical conditioning in the rat. Acta Neurobiol Exp (Wars) 69:119-128.

Klein MM, Cholvin T, Cosquer B, Salvadori A, Le Mero J, Kourouma L, Boutillier AL, Pereira de Vasconcelos A, Cassel JC (2019) Ventral midline thalamus lesion prevents persistence of new (learning-triggered) hippocampal spines, delayed neocortical spinogenesis, and spatial memory durability. Brain Struct Funct 224:16591676.

Lakens D (2013) Calculating and reporting effect sizes to facilitate cumulative science: a practical primer for t-tests and ANOVAs. Front Psychol 4:863.

Laroche S, Davis S, Jay TM (2000) Plasticity at hippocampal to prefrontal cortex synapses: dual roles in working memory and consolidation. Hippocampus 10:438-446.

Layfield DM, Patel M, Hallock H, Griffin AL (2015) Inactivation of the nucleus reuniens/rhomboid causes a delay-dependent impairment of spatial working memory. Neurobiol Learn Mem 125:163-167.

LeDoux JE (1996) The emotional brain: the mysterious underpinnings of emotional life. New York: Simon and Schuster.

Likhtik E, Stujenske JM, Topiwala MA, Harris AZ, Gordon JA (2014) Prefrontal entrainment of amygdala activity signals safety in learned fear and innate anxiety. Nat Neurosci 17:106-113.

Lin CH, Yeh SH, Lu HY, Gean PW (2003) The similarities and diversities of signal pathways leading to consolidation of conditioning and consolidation of extinction of fear memory. $J$ Neurosci 23:8310-8317.

Loureiro M, Cholvin T, Lopez J, Merienne N, Latreche A, Cosquer B, Geiger K, Kelche C, Cassel JC, Pereira de Vasconcelos A (2012) The ventral midline thalamus (reuniens and rhomboid nuclei) contributes to the persistence of spatial memory in rats. J Neurosci 32:9947-9959.

Maisson DJ, Gemzik ZM, Griffin AL (2018) Optogenetic suppression of the nucleus reuniens selectively impairs encoding during spatial working memory. Neurobiol Learn Mem 155:78-85.

Marchand A, Faugère A, Coutureau $E$, Wolff M (2014) A role for anterior thalamic nuclei in contextual fear memory. Brain Struct Funct 219:1575-1586.
Maren S, Phan KL, Liberzon I (2013) The contextual brain: implications for fear conditioning, extinction and psychopathology. Nat Rev Neurosci 14:417-428.

Martin JH (1991) Autoradiographic estimation of the extent of reversible inactivation produced by microinjection of lidocaine and muscimol in the rat. Neurosci Lett 127:160-164.

McGaugh JL (1966) Time-dependent processes in memory storage. Science 153:1351-1358.

McGaugh JL (2000) Memory-a century of consolidation. Science 287:248-251.

Mei H, Logothetis NK, Eschenko O (2018) The activity of thalamic nucleus reuniens is critical for memory retrieval, but not essential for the early phase of "off-line" consolidation. Learn Mem 25:129137.

Merlo E, Milton AL, Goozée ZY, Theobald DE, Everitt BJ (2014) Reconsolidation and extinction are dissociable and mutually exclusive processes: behavioral and molecular evidence. J Neurosci 34:2422-2431.

Milad MR, Rauch SL, Pitman RK, Quirk GJ (2006) Fear extinction in rats: implications for human brain imaging and anxiety disorders. Biol Psychol 73:61-71.

Moscovitch A, LoLordo VM (1968) Role of safety in the Pavlovian backward fear conditioning procedure. J Comp Physiol Psychol 66:673-678.

Papini MR, Bitterman ME (1990) The role of contingency in classical conditioning. Psychol Rev 97:396-403.

Pavlov IP (1927) Conditioned reflexes: an investigation of the physiological activity of the cerebral cortex. Oxford: Oxford University Press.

Phillips RG, LeDoux JE (1994) Lesions of the dorsal hippocampal formation interfere with background but not foreground contextual fear conditioning. Learn Mem 1:34-44.

Quet E, Majchrzak M, Cosquer B, Morvan T, Wolff M, Cassel JC, Pereira de Vasconcelos A, Stéphan A (2020) The reuniens and rhomboid nuclei are necessary for contextual fear memory persistence in rats. Brain Struct Funct 225:955-968.

Quirk GJ, Mueller D (2008) Neural mechanisms of extinction learning and retrieval. Neuropsychopharmacology 33:56-72.

Ramanathan KR, Ressler RL, Jin J, Maren S (2018a) Nucleus reuniens is required for encoding and retrieving precise, hippocampal-dependent contextual fear memories in rats. $\mathrm{J}$ Neurosci 38:9925-9933.

Ramanathan KR, Jin J, Giustino TF, Payne MR, Maren S (2018b) Prefrontal projections to the thalamic nucleus reuniens mediate fear extinction. Nat Commun 9:4527.

Raybuck JD, Lattal KM (2014) Bridging the interval: theory and neurobiology of trace conditioning. Behav Processes 101:103111.

Richardson JT (2011) Eta squared and partial eta squared as measures of effect size in educational research. Educ Res Rev 6:135147.

Rigoli F, Pezzulo G, Dolan RJ (2016) Prospective and Pavlovian mechanisms in aversive behaviour. Cognition 146:415-425.

Santini E, Ge H, Ren K, Peña de Ortiz S, Quirk GJ (2004) Consolidation of fear extinction requires protein synthesis in the medial prefrontal cortex. J Neurosci 24:5704-5710.

Schafe GE, Atkins CM, Swank MW, Bauer EP, Sweatt JD, LeDoux JE (2000) Activation of ERK/MAP kinase in the amygdala is required for memory consolidation of pavlovian fear conditioning. $J$ Neurosci 20:8177-8187.

Shors TJ, Beylin AV, Wood GE, Gould E (2000) The modulation of Pavlovian memory. Behav Brain Res 110:39-52.

Sierra RO, Pedraza LK, Zanona QK, Santana F, Boos FZ, Crestani AP, Haubrich J, de Oliveira Alvares L, Calcagnotto ME, Quillfeldt JA (2017) Reconsolidation-induced rescue of a remote fear memory blocked by an early cortical inhibition: involvement of the anterior cingulate cortex and the mediation by the thalamic nucleus reuniens. Hippocampus 27:596-607. 
Song C, Detert JA, Sehgal M, Moyer JR Jr (2012) Trace fear conditioning enhances synaptic and intrinsic plasticity in rat hippocampus. J Neurophysiol 107:3397-3408.

Song C, Ehlers VL, Moyer JR Jr (2015) Trace fear conditioning differentially modulates intrinsic excitability of medial prefrontal cortex-basolateral complex of amygdala projection neurons in infralimbic and prelimbic cortices. J Neurosci 35:13511-13524.

van Duuren E, van der Plasse G, van der Blom R, Joosten RN, Mulder AB, Pennartz CM, Feenstra MG (2007) Pharmacological manipulation of neuronal ensemble activity by reverse microdialysis in freely moving rats: a comparative study of the effects of tetrodotoxin, lidocaine, and muscimol. J Pharmacol Exp Ther 323:61-69.
Vertes RP (2004) Differential projections of the infralimbic and prelimbic cortex in the rat. Synapse 51:32-58.

Vertes RP, Hoover WB, Do Valle AC, Sherman A, Rodriguez JJ (2006) Efferent projections of reuniens and rhomboid nuclei of the thalamus in the rat. J Comp Neurol 499:768-796.

Viena TD, Linley SB, Vertes RP (2018) Inactivation of nucleus reuniens impairs spatial working memory and behavioral flexibility in the rat. Hippocampus 28:297-311.

Witter MP, Wouterlood FG, Naber PA, Van Haeften T (2000) Anatomical organization of the parahippocampal-hippocampal network. Ann NY Acad Sci 911:1-24.

Xu W, Südhof TC (2013) A neural circuit for memory specificity and generalization. Science 339:1290-1295. 